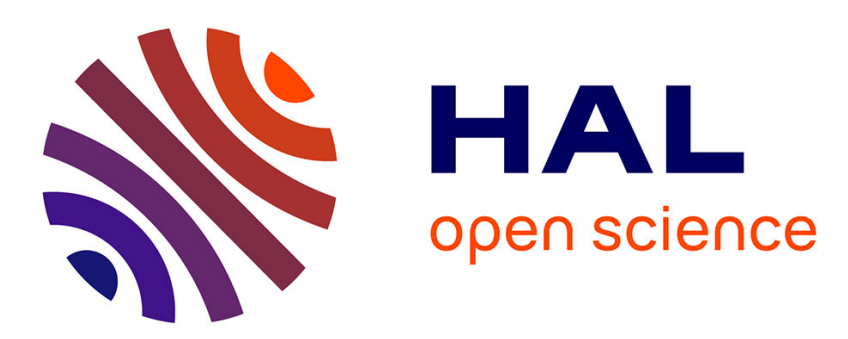

\title{
Addressing high dimensionality in reliability analysis using low-rank tensor approximations
}

\author{
K Konakli, Bruno Sudret
}

\section{To cite this version:}

K Konakli, Bruno Sudret. Addressing high dimensionality in reliability analysis using low-rank tensor approximations. European Safety and Reliability Conference ESREL 2015, Sep 2015, Zürich, Switzerland. 10.1201/b19094-541 . hal-01221021

\section{HAL Id: hal-01221021 \\ https://hal.science/hal-01221021}

Submitted on 27 Oct 2015

HAL is a multi-disciplinary open access archive for the deposit and dissemination of scientific research documents, whether they are published or not. The documents may come from teaching and research institutions in France or abroad, or from public or private research centers.
L'archive ouverte pluridisciplinaire HAL, est destinée au dépôt et à la diffusion de documents scientifiques de niveau recherche, publiés ou non, émanant des établissements d'enseignement et de recherche français ou étrangers, des laboratoires publics ou privés. 


\title{
Addressing high dimensionality in reliability analysis using low-rank tensor approximations
}

\author{
K. Konakli \& B. Sudret \\ Chair of Risk, Safety and Uncertainty Quantification \\ ETH Zürich, Switzerland
}

\begin{abstract}
Evaluation of tail probabilities in reliability analysis faces challenges in cases when complex models with high-dimensional random input are considered. To address such problems, we herein propose the use of surrogate models developed with low-rank tensor approximations. In this approach, the response quantity of interest is expressed as a sum of a few rank-one tensors. We first describe a non-intrusive method for building low-rank approximations using a greedy algorithm, which relies on the solution of minimization problems of only small size. In the sequel, we demonstrate the efficiency of meta-models built in this way in reliability applications involving the deflections of structural systems under static loads and the temperature in stationary heat conduction with spatially varying diffusion coefficient. Furthermore, we show that the proposed approach outperforms the popular meta-modeling technique of polynomial chaos expansions.
\end{abstract}

\section{INTRODUCTION}

It is nowadays common engineering practice to investigate the behavior of a system of interest through computer simulation. Because uncertainty is ubiquitous in engineering problems, the analysis of the response of a computational model must consider a range of values for the input parameters. In this context, reliability analysis aims at evaluating the probability of a response quantity exceeding a prescribed limit, the so-called failure probability.

When events with small failure probabilities are of interest, reliability analysis with the classical Monte Carlo simulation approach becomes unaffordable for complex computational models due to the large number of runs required. To address this issue, several methods have been proposed to date, each possessing its own advantages and limitations (see e.g. Morio et al. (2014)). Unfortunately, many of those methods prove inefficient in problems with high-dimensional input.

The focus herein is on reliability analysis by means of surrogate models, also known as meta-models. The latter possess similar statistical properties with the models they substitute, but have simple functional forms, thus enabling analysis of complex systems with random input. We investigate a newly emerged class of meta-models, called Low-Rank Approximations (LRA), which represent the random response as a sum of a few rank-one tensors (e.g. Nouy (2010), Chevreuil et al. (2013), Doostan et al. (2013)). Be- cause the number of unknown coefficients in LRA grows only linearly with the number of input parameters, the approach can be particularly effective in handling problems with high dimensionality.

In this paper, after a brief review of non-intrusive meta-modeling and respective error estimation techniques, we describe a method for developing LRA by building upon existing greedy approaches. We next examine reliability applications in which the number of available model evaluations for creating a metamodel is small with respect to the input dimension. In these applications, we demonstrate the superiority of LRA in comparison to the popular meta-modeling technique of polynomial chaos expansions (Xiu and Karniadakis (2002), Blatman and Sudret (2011)).

\section{NON-INTRUSIVE META-MODELING AND ERROR ESTIMATION}

We consider a computational model, $\mathcal{M}$, represented by the map

$$
\boldsymbol{X} \in \mathcal{D}_{\boldsymbol{X}} \subset \mathbb{R}^{M} \longmapsto Y=\mathcal{M}(\boldsymbol{X}) \in \mathbb{R},
$$

where $\boldsymbol{X}$ denotes the $M$-dimensional random input vector with support $\mathcal{D}_{\boldsymbol{X}}$ and $Y$ is a scalar response quantity of interest. In a general case, the above map does not have a closed analytical form and may involve a computationally expensive process.

A meta-model, $\widehat{\mathcal{M}}(\boldsymbol{X})$, is an analytical function that mimics the behavior of $\mathcal{M}(\boldsymbol{X})$. In non- 
intrusive approaches, the meta-model is developed using a set of realizations of the input vector, $\mathcal{E}=$ $\left\{\chi^{(1)}, \ldots, \chi^{(N)}\right\}$, called Experimental Design (ED), and the corresponding evaluations of the original model, $\mathcal{Y}=\left\{\mathcal{M}\left(\boldsymbol{\chi}^{(1)}\right), \ldots, \mathcal{M}\left(\boldsymbol{\chi}^{(N)}\right)\right\}$. Thus, nonintrusive meta-modeling treats the original model as a black box.

In the following, we describe measures of accuracy of the meta-model response, $\widehat{Y}=\widehat{\mathcal{M}}(\boldsymbol{X})$. To this end, we introduce the discrete $L_{2}$ semi-norm of a function $x \in \mathcal{D}_{\boldsymbol{X}} \longmapsto a(\boldsymbol{x}) \in \mathbb{R}$, given by

$$
\|a\|_{\mathcal{X}}=\left(\frac{1}{n} \sum_{i=1}^{n} a^{2}\left(\boldsymbol{x}_{i}\right)\right)^{1 / 2}
$$

where $\mathcal{X}=\left\{\boldsymbol{x}_{1}, \ldots, \boldsymbol{x}_{n}\right\} \subset \mathcal{D}_{\boldsymbol{X}}$ denotes a set of realizations of the input vector.

The generalization error, $\operatorname{Err}_{G}$, represents the mean-square error of the difference $Y-\widehat{Y}$ and can be estimated by

$$
\widehat{\operatorname{Err}}_{G}=\|\mathcal{M}-\widehat{\mathcal{M}}\|_{\mathcal{X}_{\text {val }}}^{2}
$$

where $\mathcal{X}_{\text {val }}=\left\{\boldsymbol{x}_{1}, \ldots, \boldsymbol{x}_{n_{\text {val }}}\right\}$ is a sufficiently large set of realizations of the input vector, called validation set. The estimate of the relative generalization error, $\widehat{e r r}_{G}$, is obtained by normalizing $\widehat{E r r}_{G}$ with the empirical variance of $\mathcal{Y}_{\text {val }}=\left\{\mathcal{M}\left(\boldsymbol{x}_{1}\right), \ldots, \mathcal{M}\left(\boldsymbol{x}_{n_{\text {val }}}\right)\right\}$. However, a validation set is usually not available in typical meta-modeling applications, where a large number of model evaluations is unaffordable. A different estimate that relies solely on the ED, $\mathcal{E}=$ $\left\{\chi^{(1)}, \ldots, \chi^{(N)}\right\}$, is the empirical error, $\widehat{\operatorname{Err}}_{E}$, given by

$$
\widehat{\operatorname{Err}}_{E}=\|\mathcal{M}-\widehat{\mathcal{M}}\|_{\mathcal{E}}^{2}
$$

The relative empirical error, $\widehat{e r r}_{E}$, is obtained by normalizing $\widehat{\operatorname{Err}}_{E}$ with the empirical variance of $\mathcal{Y}=\left\{\mathcal{M}\left(\boldsymbol{\chi}^{(1)}\right), \ldots, \mathcal{M}\left(\boldsymbol{\chi}^{(N)}\right)\right\}$. Unfortunately, $\widehat{\operatorname{err}}_{E}$ tends to underestimate the actual generalization error, which might be severe in cases of overfitting.

A good compromise between accuracy and computational cost in error evaluation is offered by CrossValidation (CV) techniques, which use the information contained in the ED. In $k$-fold $\mathrm{CV}$, (i) the ED is randomly partitioned into $k$ sets of approximately equal size, (ii) for $i=1, \ldots, k$, a meta-model is built considering all but the $i$-th partition and the excluded set is used to evaluate the respective generalization error, (iii) the generalization error of the meta-model built with the full ED is estimated as the average of the errors of the $k$ meta-models in (ii).

\section{LOW-RANK TENSOR APPROXIMATIONS}

\subsection{Formulation using polynomial bases}

We consider the map in Eq. (1) assuming that the components of $\boldsymbol{X}=\left\{X_{1}, \ldots, X_{M}\right\}$ are independent, with the marginal Probability Density Function (PDF) of $X_{i}$ denoted by $f_{X_{i}}$. A meta-model of $Y=\mathcal{M}(\boldsymbol{X})$ belonging to the class of LRA has the form

$$
\widehat{Y}=\sum_{l=1}^{R} b_{l}\left(\prod_{i=1}^{M} v_{l}^{(i)}\left(X_{i}\right)\right)
$$

where $v_{l}^{(i)}$ denotes a univariate function of $X_{i}, b_{l}$ is the normalizing coefficient of the rank-1 function in the parenthesis and $R$ is a small number of rank- 1 terms. By expanding $v_{l}^{(i)}\left(X_{i}\right)$ onto a polynomial basis that is orthonormal with respect to $f_{X_{i}}$, Eq. (5) takes the form

$$
\widehat{Y}=\sum_{l=1}^{R} b_{l}\left(\prod_{i=1}^{M}\left(\sum_{k=0}^{p_{i}} z_{k, l}^{(i)} P_{k}^{(i)}\left(X_{i}\right)\right)\right),
$$

where $P_{k}^{(i)}$ is the $k$-th degree univariate polynomial in the $i$-th input variable of maximum degree $p_{i}$ and $z_{k, l}^{(i)}$ is the coefficient of $P_{k}^{(i)}$ in the $l$-th rank- 1 term. We underline that the number of unknown coefficients in Eq. (6) grows only linearly with the input dimension, $M$.

For standard distributions, the associated family of orthonormal polynomials is well known. For instance, a uniform variable with support $[-1,1]$ is associated with the family of Legendre polynomials, whereas a standard normal variable is associated with the family of Hermite polynomials. Other cases can be treated through an isoprobabilistic transformation of $\boldsymbol{X}$ to a basic random vector. Cases with mutually dependent input variables can also be treated through an isoprobabilistic transformation to a vector of independent standard variables.

Note that although LRA with polynomial bases are examined in the present work, alternative basis functions may be considered in a general case.

\subsection{Construction with greedy approaches}

LRA can be efficiently built with greedy approaches, where the rank of the approximation is progressively increased and the polynomial coefficients in each dimension are sequentially updated. In this paper, we adopt the skeleton of the algorithm described in Chevreuil et al. (2013) and propose appropriate stopping criteria in the sequential updating of the polynomial coefficients.

Let $\widehat{Y}_{r}$ denote the rank- $r$ approximation of $Y=$ $\mathcal{M}(\boldsymbol{X})$, i.e.

$$
\widehat{Y}_{r}=\sum_{l=1}^{r} b_{l} w_{l}(\boldsymbol{X})
$$


with

$$
w_{l}(\boldsymbol{X})=\prod_{i=1}^{M}\left(\sum_{k=0}^{p_{i}} z_{k, l}^{(i)} P_{k}^{(i)}\left(X_{i}\right)\right) .
$$

The adopted algorithm comprises a sequence of pairs of a correction step and an updating step, so that the $r$-th correction step yields the rank-1 term $w_{r}$ and the $r$-th updating step yields the set of coefficients $b_{1}, \ldots, b_{r}$. Details are given in the sequel.

Correction step: Let $R_{r-1}$ denote the residual after the completion of the $(r-1)$-th iteration, i.e.

$$
R_{r-1}=Y-\widehat{Y}_{r-1}
$$

with $R_{0}=Y$. Based on the available experimental design, $\mathcal{E}$, the rank-1 tensor $w_{r}$ is obtained as the solution of

$$
w_{r}=\arg \min _{\omega \in \mathcal{W}}\left\|R_{r-1}-\omega\right\|_{\mathcal{E}}^{2},
$$

where $\mathcal{W}$ represents the space of rank- 1 tensors. By employing an alternated minimization algorithm, the minimization problem in Eq. (10) is substituted by a series of smaller minimization problems, each involving the coefficients along one dimension only, i.e.

$$
\boldsymbol{z}_{r}^{(j)}=\arg \min _{\boldsymbol{\zeta} \in \mathbb{R}^{p_{j}}}\left\|R_{r-1}-C^{(j)} \cdot\left(\sum_{k=0}^{p_{j}} \zeta_{k} P_{k}^{(j)}\right)\right\|_{\mathcal{E}}^{2},
$$

with

$$
C^{(j)}=\left(\prod_{i \neq j} \sum_{k=0}^{p_{i}} z_{k, r}^{(i)} P_{k}^{(i)}\right)
$$

for $j=1, \ldots, M$. Because Eq. (11) involves only $p_{j}+1$ unknowns (typically $p_{j}<20$ ), it can be easily solved using the Ordinary Least Squares (OLS) method.

The correction step is initiated by assigning arbitrary values to the unknowns and may involve several iterations over the set of dimensions. Our proposed stopping criterion combines the number of iterations, $I_{r}$, with the decrease in the relative empirical error in two successive iterations, $\Delta \widehat{e r r}_{r}$. In the latter, the relative empirical error, $\widehat{e r r}_{r}$, is obtained by normalizing

$$
\widehat{E r r}_{r}=\left\|R_{r-1}-w_{r}\right\|_{\mathcal{E}}^{2}
$$

with the empirical variance of $\mathcal{Y}=$ $\left\{\mathcal{M}\left(\chi^{(1)}\right), \ldots, \mathcal{M}\left(\chi^{(N)}\right)\right\}$, representing the set of model evaluations at the ED. Accordingly, the $r$-th correction step is terminated if either $I_{r}$ reaches a maximum allowable value, $I_{\max }$, or $\Delta \widehat{e r r}_{r}$ becomes smaller than a prescribed threshold, $\Delta \widehat{e r r}_{\min }$.
Updating step: In the $r$-th updating step, the coefficients $\boldsymbol{b}=\left\{b_{1}, \ldots, b_{r}\right\}$ are determined as the solution of

$$
\boldsymbol{b}=\arg \min _{\boldsymbol{\beta} \in \mathbb{R}^{r}}\left\|\mathcal{M}-\sum_{l=1}^{r} \beta_{l} w_{l}\right\|_{\mathcal{E}}^{2} .
$$

In the above minimization problem, $b_{r}$ is determined for the first time, whereas $\left\{b_{1}, \ldots, b_{r-1}\right\}$ are updated from their previous values. Because Eq. (14) involves only $r$ unknowns (recall that small ranks are relevant in LRA), it can be easily solved with OLS.

Note that the progressive adding of rank-1 terms results in a set of LRA of rank $\{1, \ldots, r\}$ at the $r$-th step. In the present work, we select the optimal rank $R \in\left\{1, \ldots, r_{\max }\right\}$ by using error measures based on 3 -fold CV as proposed by Chevreuil et al. (2013) (see Section 2 for details on $k$-fold $\mathrm{CV}$ ).

\subsection{Comparison to polynomial chaos expansions}

A popular approach for developing meta-models with polynomial bases is the use of Polynomial Chaos Expansions (PCE), described next. As in Section 3.1, we assume that the components of $\boldsymbol{X}$ are independent and treat the case of dependent input with an appropriate isoprobabilistic transformation.

Let $f_{\boldsymbol{X}}$ denote the PDF of $\boldsymbol{X}$, i.e.

$$
f_{\boldsymbol{X}}(\boldsymbol{X})=\prod_{i=1}^{M} f_{X_{i}}\left(X_{i}\right)
$$

Then, a PCE meta-model of $Y=\mathcal{M}(\boldsymbol{X})$ in Eq. (1) has the form (Xiu and Karniadakis (2002), Soize and Ghanem (2004))

$$
\widehat{Y}=\sum_{\boldsymbol{\alpha} \in \mathcal{A}} y_{\boldsymbol{\alpha}} \Psi_{\boldsymbol{\alpha}}(\boldsymbol{X})
$$

where $\Psi_{\alpha}$ are multivariate polynomials that are orthonormal with respect to $f_{\boldsymbol{X}}, \mathcal{A}$ is a finite subset of $\mathbb{N}^{M}$ and $y_{\boldsymbol{\alpha}}$ are the corresponding polynomial coefficients. Because of Eq. (15), the multivariate polynomial bases in Eq. (16) can be obtained by tensorization of univariate polynomials that are orthonormal with respect to the marginals $f_{X_{i}}$, i.e.

$$
\Psi_{\boldsymbol{\alpha}}(\boldsymbol{X})=\prod_{i=1}^{M} P_{\alpha_{i}}^{(i)}\left(X_{i}\right)
$$

where $\boldsymbol{\alpha}=\left\{\alpha_{1}, \ldots, \alpha_{M}\right\}$ and $P_{\alpha_{i}}^{(i)}$ is a univariate polynomial of degree $\alpha_{i}$ in the $i$-th input variable belonging to an appropriate family (see Section 3.1).

Different truncation schemes may be employed to determine the set of multi-indices $\{\boldsymbol{\alpha} \in \mathcal{A}\}$. When the maximum degree of $P_{\alpha_{i}}^{(i)}$ is set to $p_{i}$, i.e. $\mathcal{A}=$ $\left\{\boldsymbol{\alpha} \in \mathbb{N}^{M}: \alpha_{i} \leq p_{i}, i=1, \ldots, M\right\}$, the expansion 
in Eq. (16) relies on the same polynomial functions used in Eq. (6). For this case, let us compare the number of unknowns in LRA and PCE when $p_{i}=p$, $i=1, \ldots, M$. These numbers are given by $(p+1)^{M}$ in PCE and $(p+1) \cdot M \cdot R$ in LRA, disregarding redundant parameters. Accordingly, for a typical engineering problem with $M=10$, considering loworder polynomials with $p=3$ and an example low rank $R=10$, the aforementioned equations yield $1,048,576$ PCE coefficients versus a mere 400 unknowns in LRA.

A more efficient truncation scheme is the hyperbolic scheme (Blatman and Sudret 2010) defined by $\mathcal{A}=\left\{\boldsymbol{\alpha} \in \mathbb{N}^{M}:\|\boldsymbol{\alpha}\|_{q} \leq p\right\}$, with

$$
\|\boldsymbol{\alpha}\|_{q}=\left(\sum_{i=1}^{M} \alpha_{i}^{q}\right)^{1 / q}, 0<q<1 .
$$

When $q=1$, multivariate polynomials of maximum total degree $p$ are retained in the expansion. Smaller values of $q$ impose limitations to the number of terms that include interactions between two or more input variables.

When the coefficients $\boldsymbol{y}=\left\{y_{\boldsymbol{\alpha}}, \boldsymbol{\alpha} \in \mathcal{A}\right\}$ are determined with a regression approach, one needs to solve the minimization problem

$$
\boldsymbol{y}=\arg \min _{\boldsymbol{v} \in \mathbb{R}^{\operatorname{card} \mathcal{A}}}\left\|\mathcal{M}-\sum_{\boldsymbol{\alpha} \in \mathcal{A}} v_{\boldsymbol{\alpha}} \Psi_{\boldsymbol{\alpha}}\right\|_{\mathcal{E}}^{2} .
$$

Even by employing a hyperbolic truncation scheme, the number of unknowns in Eq. (19) can be very large in high-dimensional problems, requiring ED of unaffordable size. Note that contrary to LRA, where a greedy construction allows computation of the polynomial coefficients in each direction separately, the entire set of PCE coefficients is determined from a single minimization problem. To improve efficiency in the latter, one may substitute Eq. (19) with a respective regularized problem. By penalizing the $L_{1}$ norm of $\boldsymbol{y}$, the Least Angle Regression (LAR) method (Efron et al. 2004) disregards insignificant terms from the set of predictors, thus yielding sparse PCE. A variation proposed by Blatman and Sudret (2011) under the name hybrid LAR consists in using the LAR method to determine the best set of predictors and then, computing the PCE coefficients with OLS.

\section{EXAMPLE APPLICATIONS}

\subsection{Beam deflection}

In the first example, we investigate the reliability of a simply supported beam under a concentrated load at the mid-span with respect to the mid-span deflection. The model input, $\boldsymbol{X}$, comprises $M=5$ independent variables: the width and height of the rectangular cross section, respectively denoted by $b$ and $h$; the length, $L$; the Young's modulus, $E$; and the load, $P$. The distributions of the input variables are listed in Table 1. The mid-span deflection is obtained through basic structural mechanics as $u=P L^{3} / 4 E b h^{3}$. The probability of failure is defined by $P_{f}=\mathbb{P}\left(u \geq u_{\text {lim }}\right)$, where $u_{\text {lim }}$ denotes a maximum allowable deflection. Due to the problem setup ( $u$ is a product of lognormal random variables), $P_{f}$ can be computed analytically.

Table 1: Simply-supported beam: Distributions of input random variables.

\begin{tabular}{cccc}
\hline Variable & Distribution & mean & CoV \\
\hline$b(\mathrm{~m})$ & Lognormal & 0.15 & 0.05 \\
$h(\mathrm{~m})$ & Lognormal & 0.3 & 0.05 \\
$L(\mathrm{~m})$ & Lognormal & 5 & 0.01 \\
$E(\mathrm{MPa})$ & Lognormal & 30,000 & 0.15 \\
$P(\mathrm{KN})$ & Lognormal & 10 & 0.20 \\
\hline
\end{tabular}

We obtain failure probability estimates, $\widehat{P}_{f}$, for different deflection thresholds using a Monte Carlo Simulation (MCS) approach, in which the actual model $u(\boldsymbol{X})$ is replaced by a meta-model $\widehat{u}(\boldsymbol{X})$. In addition to LRA, we consider meta-models built with PCE and assess their comparative accuracy. We use two ED, of sizes $N=30$ and $N=50$, both obtained with Sobol pseudo-random sequences. In the LRA approach, we use a common polynomial degree $p_{1}=\ldots=p_{5}=p$, selected with 3 -fold CV. After preliminary investigations, we set $I_{\max }=50$ and $\Delta \widehat{e r r}_{\min }=10^{-8}$ (see Konakli and Sudret (2015)). In the PCE approach, we determine the candidate basis using a hyperbolic truncation scheme and develop sparse expansions by computing the coefficients with hybrid LAR. Optimal values of $\{p, q\}$ are selected by means of the leaveone-out error (see Blatman and Sudret (2011) for details). The software UQLab (Marelli and Sudret 2014) is used for building the PCE meta-models.

For $u_{\text {lim }} \in[4,9] \mathrm{mm}$, Figure 1 compares the reliability indices, $\widehat{\beta}=\Phi^{-1}\left(\widehat{P}_{f}\right)$, obtained with the LRA and PCE meta-models to the corresponding reference reliability index, $\beta=\Phi^{-1}\left(P_{f}\right)$, representing the analytical solution. For $N=30$, the estimates obtained with LRA are fairly close to $\beta$, especially for the smaller deflection thresholds, whereas the estimates obtained with PCE are overall highly inaccurate (note that no failure points are sampled for $u_{l i m}=9 \mathrm{~mm}$ ). For $N=50$, the LRA-based estimates of $\beta$ are excellent in the entire range examined, whereas the PCEbased estimates remain poor for $u_{l i m}>6 \mathrm{~mm}$. To gain further insight, in Figure 2, we compare the analytical PDF of the response, $f_{U}$, to the respective Kernel Density Estimates (KDE), $\widehat{f}_{U}$, obtained with the LRA and PCE meta-models for the two ED. By using a log-scale in the vertical axes, we clearly demonstrate the superiority of LRA in estimating the tails of the response distribution. The remarkably good performance of LRA herein can be explained by the rank-1 structure of the considered response function. 

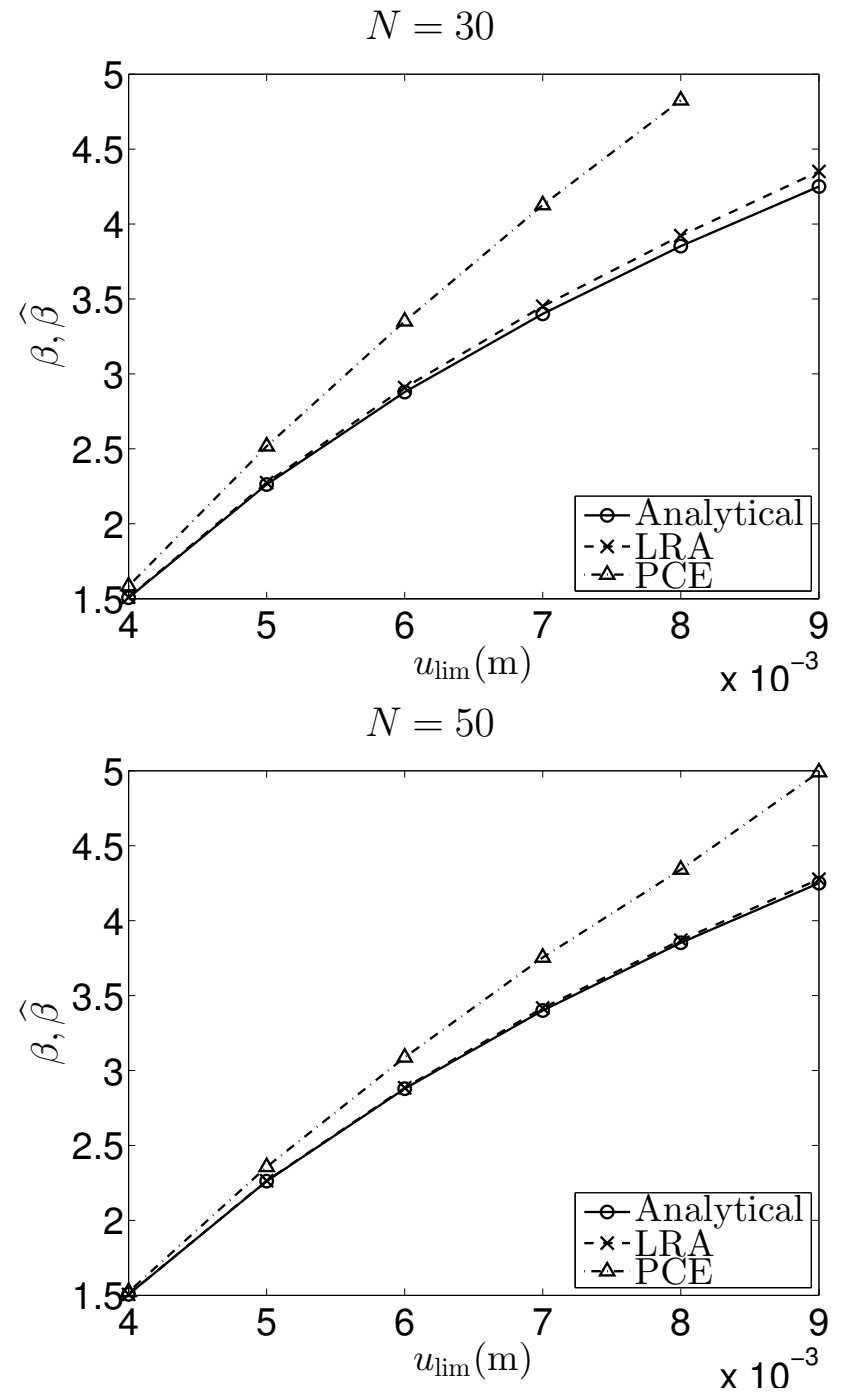

Figure 1: Reliability indices for beam deflection.

\subsection{Truss deflection}

In this example, we consider the reliability of the truss shown in Figure 3, with respect to the mid-span deflection, $u$. The model input, $\boldsymbol{X}$, comprises $M=10$ independent variables: the vertical loads, $P_{1}, \ldots, P_{6}$; the cross-sectional area and Young's modulus of the horizontal bars, $A_{1}$ and $E_{1}$, respectively; and the cross-sectional area and Young's modulus of the vertical bars, $A_{2}$ and $E_{2}$, respectively. The distributions of the input variables are listed in Table 2. The deflection, $u$, is computed with a finite-element analysis code.

Table 2: Truss structure: Distributions of input random variables.

\begin{tabular}{cccc}
\hline Variable & Distribution & mean & CoV \\
\hline$A_{1}(m)$ & Lognormal & 0.002 & 0.10 \\
$A_{2}(m)$ & Lognormal & 0.001 & 0.10 \\
$E_{1}, E_{2}\left(N / m^{2}\right)$ & Lognormal & $2.1 \mathrm{e} 11$ & 0.10 \\
$P_{1}, \ldots, P_{6}(N)$ & Gumbel & $5 \mathrm{e} 4$ & 0.15 \\
\hline
\end{tabular}

As in the previous example, we use LRA metamodels to obtain MCS estimates of the reliability indices for different deflection thresholds. We consider again a common polynomial degree $p_{1}=\ldots=p_{10}=$ $p$, selected with 3-fold CV. After preliminary investigations, we set $I_{\max }=50$ and $\Delta \widehat{e r r}_{\min }=10^{-5}$ (Kon-

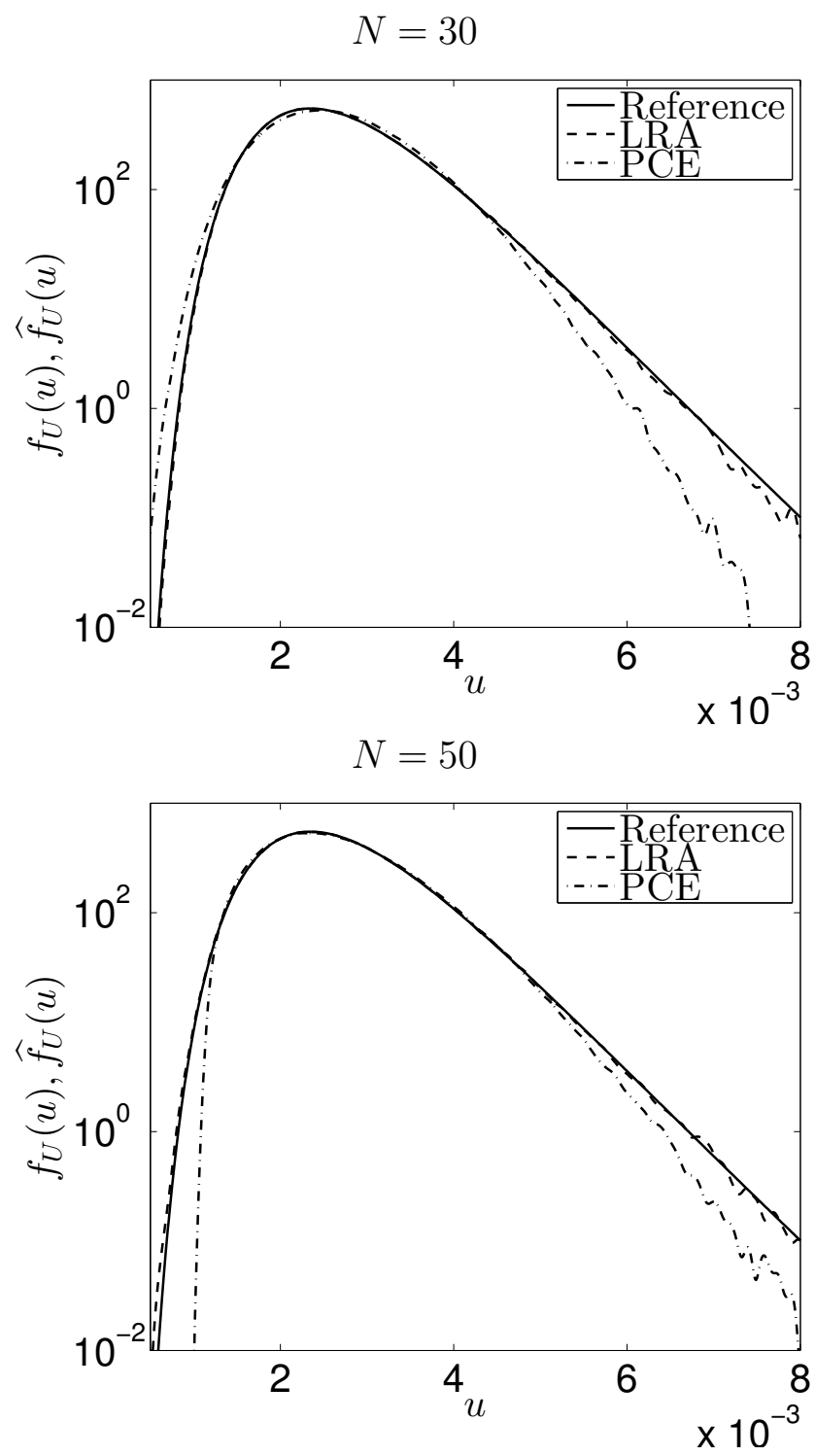

Figure 2: Probability density function of beam deflection.

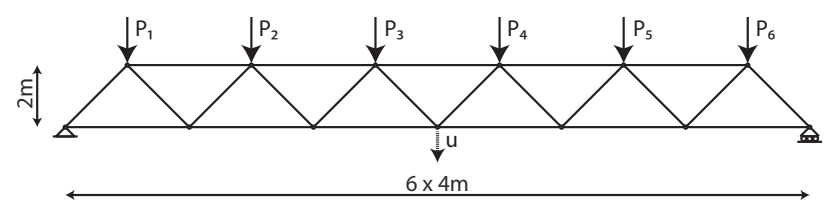

Figure 3: Truss structure.

akli and Sudret 2015). The reliability indices obtained with LRA using an ED of size $N=50$ are compared to respective values obtained with (i) the SecondOrder Reliability Method (SORM) and (ii) Importance Sampling (see e.g. Morio et al. (2014) for a description of these methods). In the IS approach, we utilize the results of a previous analysis with the FirstOrder Reliability Method (FORM) and sequentially add samples of size $N_{I S}=100$ until the coefficient of variation of the estimated failure probability becomes smaller than 0.10. The SORM- and IS-based failure probabilities are computed with the software UQLab (Marelli \& Sudret 2014).

Figure 4 compares the reliability indices obtained with the different approaches for $u_{l i m} \in[10,15] \mathrm{cm}$. We observe that the LRA-based estimates of $P_{f}$ are very close to the SORM and IS results. To highlight the efficiency of the LRA approach, in Table 3, 
we list the number of model evaluations required by SORM and IS to compute the failure probability for each threshold. The given number for IS includes the model evaluations required to obtain the FORM estimate. Note that with the LRA approach, we estimate $\widehat{P}_{f}$ values similar to those obtained with SORM and IS, while relying on a much smaller number of model evaluations. We underline that once the LRA metamodel is built, the failure probability for any threshold can be estimated without any additional model evaluations, whereas a new set of model evaluations for each threshold is required by the FORM, SORM and IS techniques.

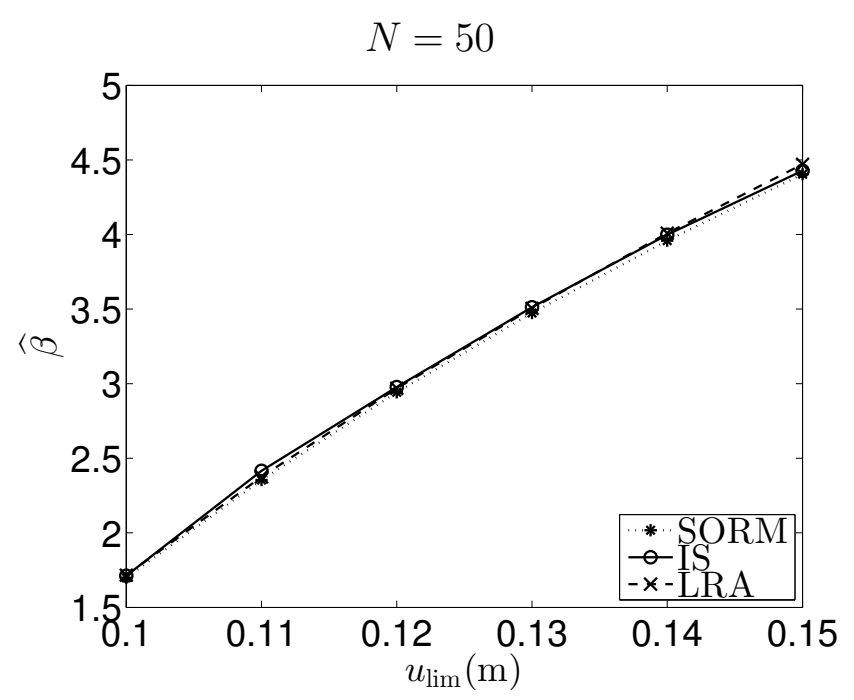

Figure 4: Reliability indices for truss deflection.

Table 3: Number of model evaluations in computing the SORM and IS estimates of the truss failure probability.

\begin{tabular}{ccc}
\hline$u_{\text {lim }}(\mathrm{m})$ & SORM & IS \\
\hline 0.10 & 387 & 475 \\
0.11 & 365 & 553 \\
0.12 & 372 & 560 \\
0.13 & 367 & 755 \\
0.14 & 379 & 867 \\
0.15 & 391 & 979 \\
\hline
\end{tabular}

\subsection{Heat conduction with spatially varying diffusion coefficient}

We consider a two-dimensional stationary heatconduction problem defined on the square domain $D=(-0.5,0.5) \times(-0.5,0.5)$ shown in Figure 5, where the temperature field $T(\boldsymbol{z}), \boldsymbol{z} \in D$, is described by the partial differential equation

$$
-\nabla(\kappa(\boldsymbol{z}) \nabla T(\boldsymbol{z}))=500 I_{A}(\boldsymbol{z}),
$$

with boundary conditions $T=0$ on the top boundary and $\nabla T \cdot \boldsymbol{n}=0$ on the left, right and bottom boundaries, where $\boldsymbol{n}$ denotes the vector normal to the boundary. In Eq. (20), $A=(0.2,0.3) \times(0.2,0.3)$ is a square domain within $D$ (see Figure 5 ) and $I_{A}$ is the indicator function equal to 1 if $\boldsymbol{z} \in A$ and 0 otherwise. The diffusion coefficient, $\kappa(\boldsymbol{z})$, is a lognormal random field defined as

$$
\kappa(\boldsymbol{z})=\exp \left[a_{\kappa}+b_{\kappa} g(\boldsymbol{z})\right]
$$

where $g(\boldsymbol{z})$ denotes a standard Gaussian random field with autocorrelation function

$$
\rho\left(\boldsymbol{z}, \boldsymbol{z}^{\prime}\right)=\exp \left(-\left\|\boldsymbol{z}-\boldsymbol{z}^{\prime}\right\|^{2} / \ell^{2}\right) .
$$

In Eq. (21), the parameters $a_{\kappa}$ and $b_{\kappa}$ are such that the mean and standard deviation of $\kappa$ are $\mu_{\kappa}=1$ and $\sigma_{\kappa}=0.3$, respectively, while in Eq. (22), $\ell=0.2$.

Using the Expansion Optimal Linear Estimation (EOLE) method (Li and Der Kiureghian 1993), the random field $g(\boldsymbol{z})$ is approximated by

$$
\widehat{g}(\boldsymbol{z})=\sum_{i=1}^{M} \frac{\xi_{i}}{\sqrt{l_{i}}} \boldsymbol{\phi}_{i}^{\mathrm{T}} \boldsymbol{C}_{\boldsymbol{z} \boldsymbol{\zeta}}
$$

In the above equation, $\left\{\xi_{1}, \ldots, \xi_{M}\right\}$ are independent standard normal variables; $\boldsymbol{C}_{\boldsymbol{z} \zeta}$ is a vector with elements $\boldsymbol{C}_{\boldsymbol{z} \boldsymbol{\zeta}}^{(k)}=\rho\left(\boldsymbol{z}, \boldsymbol{\zeta}_{k}\right)$, where $\left\{\boldsymbol{\zeta}_{1}, \ldots, \boldsymbol{\zeta}_{n}\right\}$ are the points of an appropriately defined grid in $D$; and $\left(l_{i}, \phi_{i}\right)$ are the eigenvalues and eigenvectors of the correlation matrix $\boldsymbol{C}_{\boldsymbol{\zeta} \zeta}$ with elements $\boldsymbol{C}_{\boldsymbol{\zeta}}^{(k, l)}=$ $\rho\left(\boldsymbol{\zeta}_{k}, \boldsymbol{\zeta}_{l}\right)$, where $k, l=1, \ldots, n$. We select $M=53$ in order to satisfy the condition

$$
\sum_{i=1}^{M} l_{i} / \sum_{i=1}^{n} l_{i} \geq 0.99
$$

The response quantity of interest in the following reliability analysis is the average temperature in the square domain $B=(-0.3,-0.2) \times(-0.3,-0.2)$ (see Figure 5), denoted by $\widetilde{T}$. For a given realization of $\left\{\xi_{1}, \ldots, \xi_{M}\right\}$, the "exact" model response is obtained with an in-house finite-element analysis code developed in Matlab environment. The employed finiteelement discretization with triangular Delaunay elements is shown in Figure 5. Figure 6 shows an example realization of the temperature field over the entire domain.

As before, we assess the comparative accuracy of LRA and PCE in estimating failure probabilities with MCS. We build the meta-models as described in Section 4.1, using two ED of sizes $N=200$ and $N=500$. After preliminary investigations, we set $I_{\max }=50$ and $\Delta \widehat{e r r}_{\min }=10^{-6}$ in the LRA algorithm. Reference values of the reliability indices are obtained with MCS based on evaluations of the "exact" model at an input sample consisting of $10^{4}$ points. Note that for the high input dimension of this problem, the FORM and SORM approaches are ineffective.

For varying thresholds of $\widetilde{T}, \widetilde{T}_{\text {lim }} \in[1.2,1.7]{ }^{\circ} \mathrm{C}$, Figure 7 compares the reliability indices obtained 


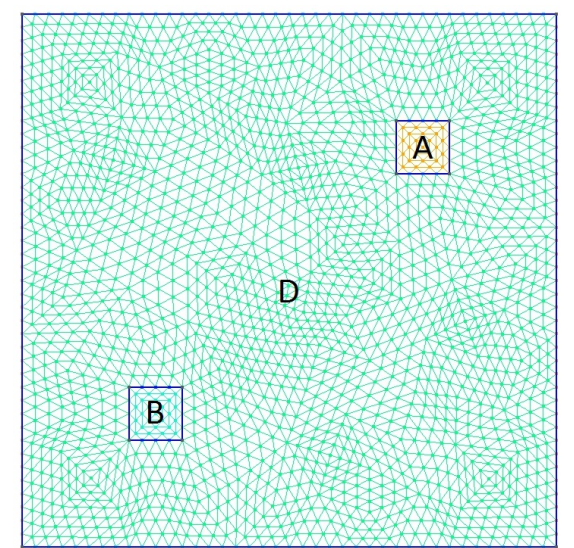

Figure 5: Domain and finite element mesh in heat-conduction problem.

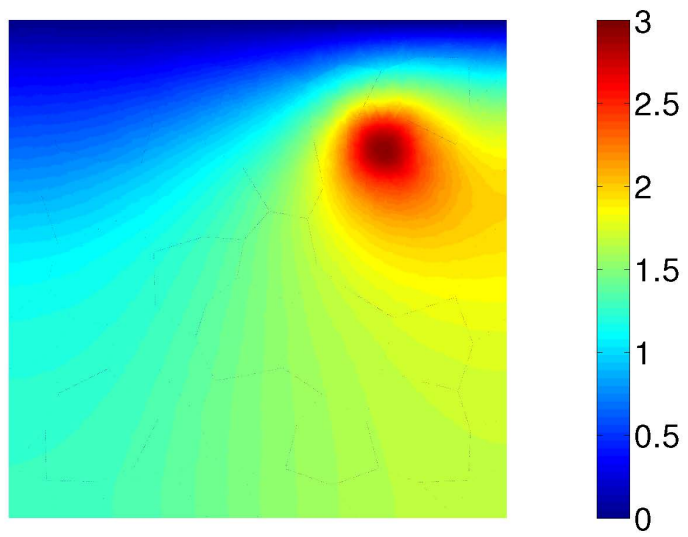

Figure 6: Example realization of temperature field in heatconduction problem.

with the LRA and PCE meta-models to their respective reference values. The figure demonstrates that for each of the considered ED, the LRA-based estimates are superior to the PCE-based ones. An ED of size $N=200$ proves sufficient to obtain fair estimates of the reference reliability indices with the LRA approach, whereas the same ED proves inadequate for estimating the higher reliability indices with the PCE approach. For $N=500$, the PCE-based estimates become acceptable, whereas the LRA-based estimates become excellent over the entire range of considered thresholds. To gain insight, in Figure 8, we compare the KDE-based PDF of $\widetilde{T}$ using the LRA and PCE meta-models for each ED to the KDE-based PDF obtained with the same set of model evaluations used to compute the reference reliability indices. The use of a logarithmic scale in the vertical axes highlights the superiority of the LRA approach in approximating the tails of the response distributions, particularly for the smaller ED.

\section{CONCLUSIONS}

For models with high-dimensional input, commonly used methods of reliability analysis may be ineffective. As a remedy, we herein propose the use of metamodels developed with Low-Rank Approximations
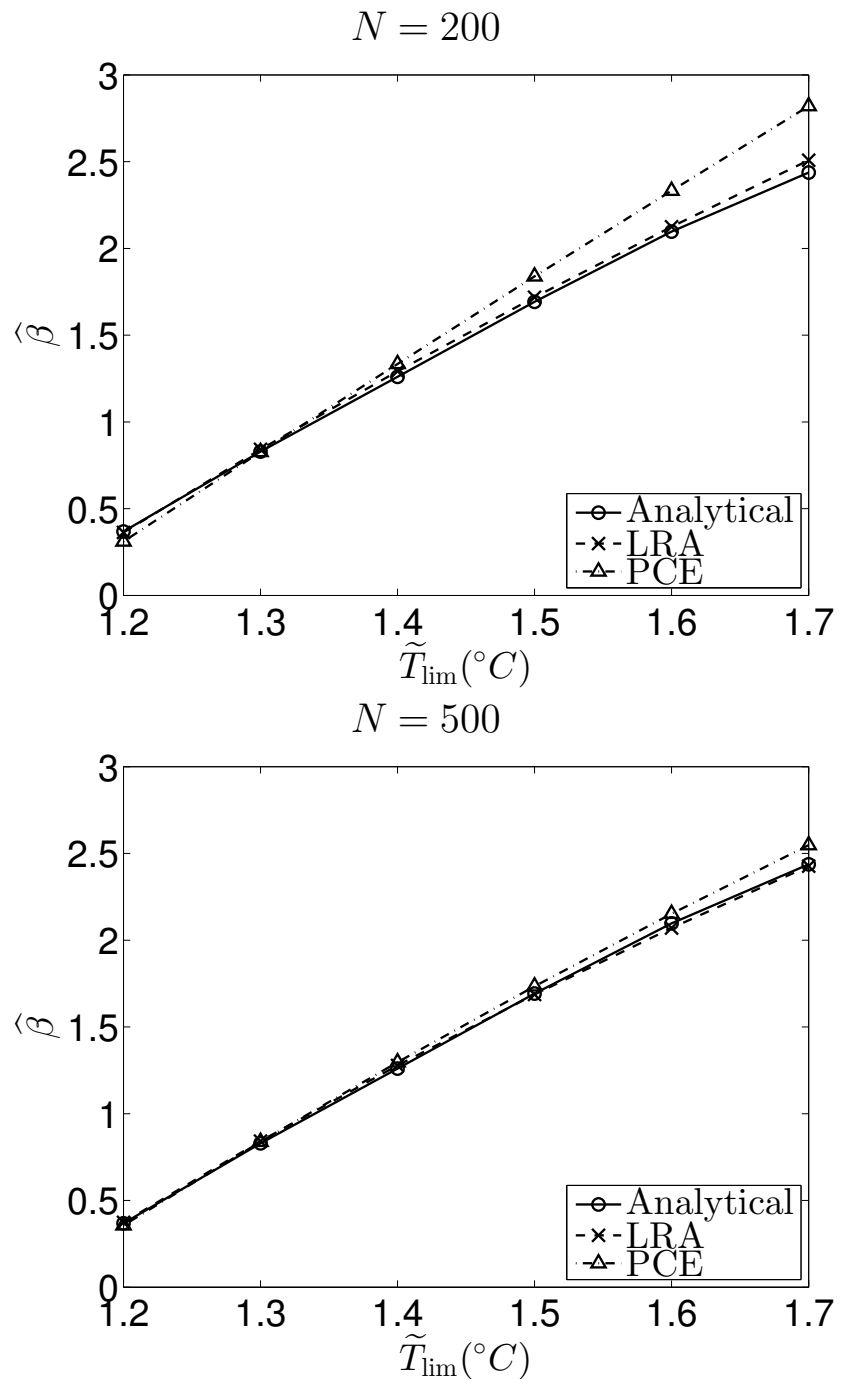

Figure 7: Reliability indices for temperature in heat-conduction problem.

(LRA). Following the description of an algorithm for building LRA non-intrusively, we demonstrate the efficiency of the approach in reliability applications, where models with different dimensionality are considered. The applications involve the deflections of a beam and a truss, both subjected to static loads, and the temperature in heat conduction with the diffusion coefficient described by a random field. In the same applications, we show that LRA outperform the popular meta-modeling technique of polynomial chaos expansions for cases when the size of the experimental design is small with respect to the input dimension.

\section{REFERENCES}

Blatman, G. \& B. Sudret (2010). An adaptive algorithm to build up sparse polynomial chaos expansions for stochastic finite element analysis. Prob. Eng. Mech. 25(2), 183-197.

Blatman, G. \& B. Sudret (2011). Adaptive sparse polynomial chaos expansion based on least angle regression. J. Comput. Phys. 230, 2345-2367.

Chevreuil, M., R. Lebrun, A. Nouy, \& P. Rai (2013). A leastsquares method for sparse low rank approximation of multivariate functions. arXiv preprint arXiv:1305.0030.

Chevreuil, M., P. Rai, \& A. Nouy (2013). Sampling based tensor approximation method for uncertainty propagation. In Proceedings of the 11th International Conference on Structural Safety and Reliability, New York, June 2013. 


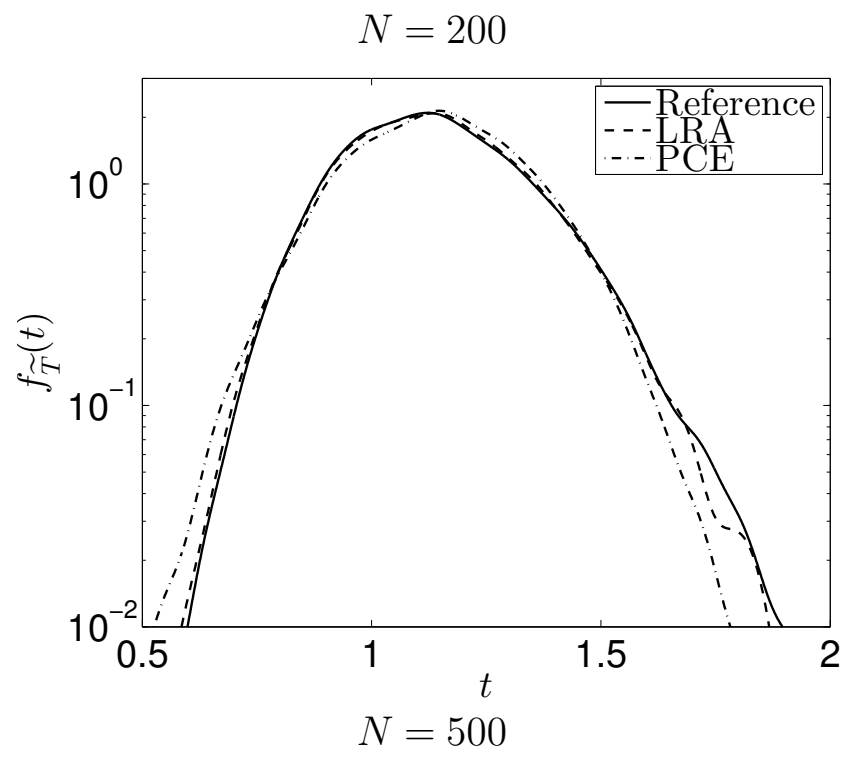

mial chaos for stochastic differential equations. SIAM Journal on Scientific Computing 24(2), 619-644.

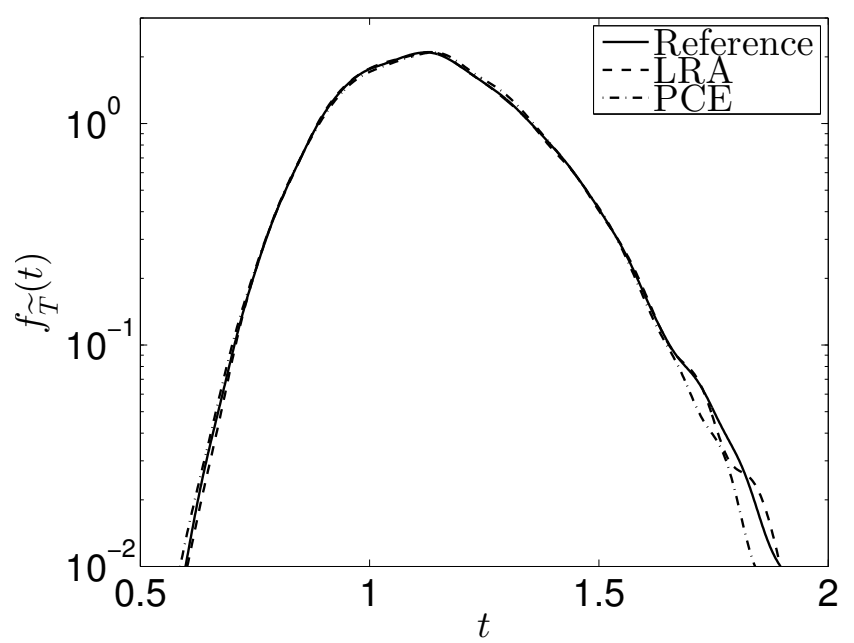

Figure 8: Probability density function of temperature in heatconduction problem.

Doostan, A., A. Validi, \& G. Iaccarino (2013). Non-intrusive low-rank separated approximation of high-dimensional stochastic models. Comput. Methods Appl. Mech. Eng. 263, 42-55.

Efron, B., T. Hastie, I. Johnstone, \& R. Tibshirani (2004). Least angle regression. Annals of Statistics 32, 407-499.

Konakli, K. \& B. Sudret (2015). Low-rank tensor approximations for reliability analysis. In Proceedings of the 12th International Conference on Applications of Statistics and Probability in Civil Engineering, Vancouver, Canada, July 2015.

Li, C.-C. \& A. Der Kiureghian (1993). Optimal discretization of random fields. Journal of Engineering Mechanics 119(6), 1136-1154.

Marelli, S. \& B. Sudret (2014). UQLab: a framework for uncertainty quantification in MATLAB. In Proc. 2nd Int. Conf. on Vulnerability, Risk Analysis and Management (ICVRAM2014), Liverpool, United Kingdom.

Morio, J., M. Balesdent, D. Jacquemart, \& C. Vergé (2014). A survey of rare event simulation methods for static inputoutput models. Simulation Modelling Practice and Theory $49,287-304$.

Nouy, A. (2010). Proper generalized decompositions and separated representations for the numerical solution of high dimensional stochastic problems. Archives of Computational Methods in Engineering 17, 403-434.

Soize, C. \& R. Ghanem (2004). Physical systems with random uncertainties: chaos representations with arbitrary probability measure. SIAM Journal on Scientific Computing 26(2), 395-410.

Xiu, D. \& G. E. Karniadakis (2002). The Wiener-Askey polyno- 